\title{
Synthetic semi-supervised learning in imbalanced domains: Constructing a model for donor-recipient matching in liver transplantation
}

\author{
M. Pérez-Ortiz ${ }^{\mathrm{a}, *}$, P.A. Gutiérrez ${ }^{\mathrm{b}}$, M. D. Ayllón-Terán ${ }^{\mathrm{c}}$, N. Heaton ${ }^{\mathrm{d}}$, R. Ciria ${ }^{\mathrm{c}}$, J. Briceño ${ }^{\mathrm{c}}$, C. Hervás-Martínez ${ }^{\mathrm{b}}$ \\ ${ }^{a}$ Department of Quantitative Methods, Universidad Loyola Andaluía, Córdoba, Spain \\ ${ }^{b}$ Department of Computer Science and Numerical Analysis, University of Córdoba, Córdoba, Spain \\ ${ }^{c}$ Liver Transplantation Unit, Reina Sofía Hospital, Córdoba, Spain \\ ${ }^{d}$ Liver Transplantation Unit, King's College, London, United Kingdom
}

\begin{abstract}
Liver transplantation is a promising and widely-accepted treatment for patients with terminal liver disease. However, transplantation is restricted by the lack of suitable donors, resulting in significant waiting list deaths. This paper proposes a novel donor-recipient allocation system that uses machine learning to predict graft survival after transplantation using a dataset comprised of donorrecipient pairs from the King's College Hospital (United Kingdom). The main novelty of the system is that it tackles the imbalanced nature of the dataset by considering semi-supervised learning, analysing its potential for obtaining more robust and equitable models in liver transplantation. We propose two different sources of unsupervised data for this specific problem (recent transplants and virtual donor-recipient pairs) and two methods for using these data during model construction (a semi-supervised algorithm and a label propagation scheme). The virtual pairs and the label propagation method are shown to alleviate the imbalanced distribution. The results of our experiments show that the use of synthetic and real unsupervised information helps to improve and stabilise the performance of the model and leads to fairer decisions with respect to the use of only supervised data. Moreover, the best model is combined with the Model for End-stage Liver Disease score (MELD), which is at the moment the most popular assignation methodology worldwide. By doing this, our decision-support system considers both the compatibility of the donor and the recipient (by our prediction system) and the recipient severity (via the MELD score), supporting then the principles of fairness and benefit.
\end{abstract}

Keywords:

Liver transplantation, Transplant recipient, Survival analysis, Machine learning, Support vector machines, Semi-supervised learning, Imbalanced classification

\section{Introduction}

In the last decades, new trends in biomedicine have used machine learning as a useful tool for a wide range of problems, resulting in remarkable applications for science (Tseng and Liao, 2009; Su and Wu, 2011). Nowadays, liver transplantation represents a promising and accepted treatment for patients with end-stage liver disease. Nevertheless, transplantation is greatly hampered by the unavailability of suitable liver donors. Several methods have been developed and applied to find a better allocation system, able to prioritise recipients on the waiting list.

The first developed system for this purpose is the Donor Risk Index (DRI) (Feng et al., 2006), that establishes the quantitative risk of the transplant considering only donor information. On the other hand, the Model for End-stage Liver Disease (MELD) (Kamath and Kim, 2007) is a widely validated methodology, globally considered as the cornerstone of the current policy for transplant allocation. This index is based on the "sickest-first" principle and uses only information of the recipient. Figure 1

${ }^{*}$ Corresponding author at: Department of Quantitative Methods, Universidad Loyola Andaluía, Third Building, C/ Escritor Castilla Aguayo 4, 14004 Córdoba, Spain. E-mail addresses: mariaperez@uloyola.es, pagutierrez@uco.es, chervas@uco.es graphically represents the current process for organ allocation (figure restructured from (Schaubel et al., 2009)). Note that computational models are used for this purpose as a decision support system. As previously mentioned, donors are generally assigned to the candidates at greatest-risk (computed by the MELD score), a policy that does not allow the transplant team to do the matching according to the principles of fairness and survival benefit (i.e. pre-transplant and post-transplant mortality), which could lead to a risk of unconscious gaming when trying to match marginal donors to urgent candidates (Pérez-Ortiz et al., 2014). The method proposed here for organ allocation seeks to minimize futile liver transplantation, giving primary attention to patients with the best predicted lifetime gained due to transplantation. Under a survival benefit model, an allocated graft goes to the patient with the greatest difference between the predicted post-transplant life-time and the predicted waiting list lifetime for this specific donor.

As shown in previous research, there are different donor characteristics which result in an increased risk and/or graft losses (Busuttil and Tanaka, 2003). These risks and characteristics should be carefully considered and included in the decision support system, since the combination of several of these risk factors can result in graft loss (Briceño et al., 2000). More- 


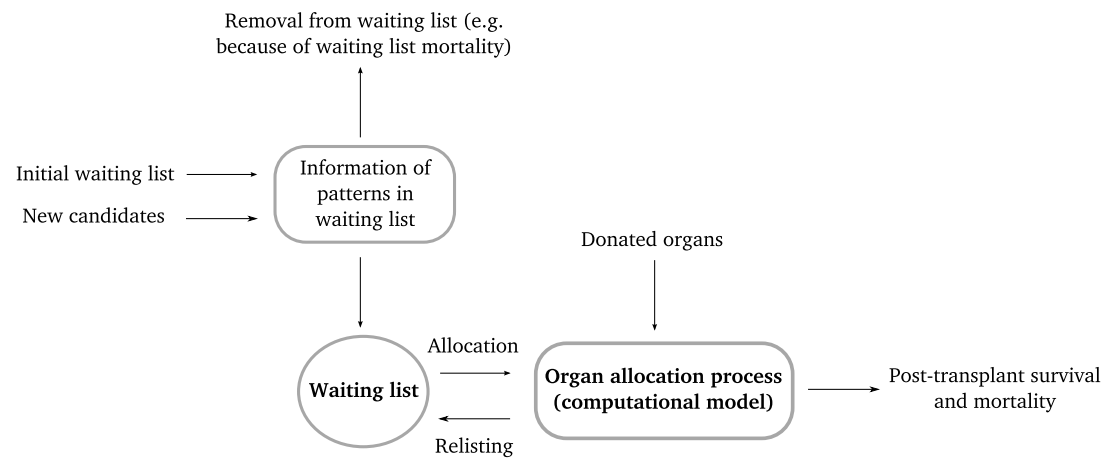

Figure 1: Graphic representing the organ allocation process.

over, it has been noted that there are some factors (concerning both the donor and the recipient) that influence the outcome of transplantation to a great extent (Pérez-Ortiz et al., 2014). Because of this, these first approaches cannot be considered good predictors of graft failure after transplantation, since they only take into consideration either characteristics of donors or recipients (but not both). New methods have emerged in the last years to deal with these issues. Rana et al. (2008) devised a scoring system (named SOFT) that predicts survival 3 months after liver transplantation, to complement MELD waiting list mortality by making use of both donor and recipient characteristics. Dutkowski et al. (2011) recently proposed a balance of risk (BAR) score based on donor and recipient characteristics. Finally, in (Cruz-Ramírez et al., 2012; Briceño et al., 2014), a rule-based system was developed to determine graft survival one year after the transplant using data from different Spanish liver transplantation units, showing that the use of machine learning substantially improves the prediction capabilities of all previous approaches. In this case, the input of this rule-based system was the response of two artificial neural networks trained with donor, recipient and transplant organ characteristics (all of these sources of information being used in this paper) and using evolutionary computation. One of the main limitations of the approaches developed in (Cruz-Ramírez et al., 2012; Briceño et al., 2014) is that, in order to approach the imbalanced nature of the data, specific fitness functions are applied for tuning the neural network weights and structure through the use of multi-objective evolutionary algorithms, thus the corresponding computational cost is very high.

Although the good performance of machine learning methods has been assessed for donor-recipient matching, the imbalanced nature of the data is still a handicap, as the results for the minority class tend to be worse (with respect to the majority one). Class imbalance is indeed one of the most common problems found in medical applications (and also in machine learning in general (Maalouf and Siddiqi, 2014; He and García, 2009)), where one or several classes have a much lower prior probability in the training set (in the context of this paper the less frequent class is graft loss, although correctly predicting a failure is the main objective). This fact needs to be taken into account in the model construction phase, because, otherwise, one could obtain accurate but trivial models (i.e. that always predict the majority class). The approaches developed over the years for tackling the class imbalance problem can be categorised in two groups: sampling (He and García, 2009; Chawla et al., 2002) and algorithmic approaches (Chang and Lin, 2011). Sampling concerns those methods that rely on a modification of the dataset (e.g. by over-sampling new data or by undersampling) and algorithmic approaches modify the classifier (e.g. using a cost-sensitive method). Although both oversampling and under-sampling approaches have been shown to improve classifier performance over imbalanced datasets, it has been shown in different studies that over-sampling is more useful than under-sampling (Japkowicz and Stephen, 2002a), specially for highly imbalanced and small datasets. Concerning cost-sensitive approaches, several works have shown that a replication of data or an imposition of higher weights for some patterns could result in over-fitting (Galar et al., 2012; PérezOrtiz et al., 2016).

In this paper, our main focus is to develop different strategies to improve the classification of the minority class, based on simpler implementations than the ones used in previous research (Cruz-Ramírez et al., 2012; Briceño et al., 2014). At the same time, we evaluate the applicability of this strategy to other transplant units, by considering a liver transplant dataset obtained from the King's College Hospital in the United Kingdom. Specifically, we tackle the imbalanced nature of the dataset by taking advantage of virtual donor-recipient pairs to improve the accuracy on the minority class. This new perspective for alleviating the imbalance problem in transplantation datasets is based on the use of semi-supervised learning. Important unsupervised information is available at the hospital and can be introduced during model construction by two ideas: exploiting very recent transplants (those whose follow-up time is not completed) and generating non-real or virtual matchings from other pairs that have been already transplanted (i.e. using potential organ transplantations that could have occurred in the past but did not). Most existing semi-supervised learning methods assume a balance between negative and positive samples in both the labelled and unlabelled data (Li et al., 2011). Unfortunately, the issue of semi-supervised learning with imbalanced data has been barely studied in the literature (Li et al., 2011; Ma et al., 2011; Huang and Kecman, 2004), only mainly from the under-sampling and ensemble points of view. The proposed un- 
supervised data generation (virtual or real) can reduce the bias of the obtained classifiers towards the majority class. Although the number of successful techniques to approach class imbalance is large, we compare our proposals to two well-known ideas: over-sampling and cost-sensitive learning (Chawla et al., 2002; Zhou, 2013), which are also the techniques that have been seen to perform better with Support Vector Machines (López et al., 2013) (the classification paradigm used in this paper).

In summary, this paper studies different hypotheses concerning imbalanced data in semi-supervised scenarios: 1) whether a large amount of unlabelled data could help tackling the imbalanced classification problem, 2) whether the ratio of positive/negative unlabelled patterns affects the results of the semisupervised method, and 3) whether it is possible to successfully label unlabelled data and balance the class distribution. Therefore, apart from considering two sources of unlabelled data, this paper also explores two approaches for using these data during model construction (a semi-supervised algorithm and a label propagation scheme). Concerning the application to transplantation data, our contributions are also noteworthy: 1) The analysis of our results with a new set of data obtained from the King's College hospital, 2) the inclusion of expert knowledge in the system (e.g. using extended donors), 3) a thorough analysis of the best model, including a study of the most important variables and a simulation of the system in a controlled environment. The performance reported in this paper shows that the use of unsupervised data (both synthetic and real) generally results in a significant improvement of the models and leads to very promising performance when predicting survival after 3 and 12 months post-transplant.

The paper is organised as follows: Section II shows a description of the semi-supervised methodology used in this work; Section III thoroughly explains the constructed dataset and the experiments to be performed; Section IV presents and analyses the results of the above-mentioned experiments; in Section V, a simulation of the proposal is performed; and finally, Section VI outlines some conclusions and future work.

\section{Methodology}

The recently coined term weak supervision (HernándezGonzález et al., 2016) refers to those machine learning problems where the labelling information is not as accessible as in the fully-supervised problem (where, in the case of classification, a label is associated to each pattern). Semi-supervised learning (i.e. learning from both labelled and unlabelled observations) is an example that has been the focus of many machine learning researchers in the past years. This is because, in many real-world applications, obtaining labelled patterns can be a challenging task, while unlabelled examples might be available with little or no cost. The main idea behind semisupervised learning is to take advantage from unlabelled data when constructing a classifier. These learning approaches have been empirically and theoretically studied in the literature and represent a suitable solution for such circumstances. Semisupervised learning has being studied mainly for binary classification (Cai et al., 2007; Cohen et al., 2004) and regression
(Zhu, 2005). This paper tackles the use of semi-supervised data for constructing a donor-recipient matching in liver transplantation, where the data distribution is highly imbalanced.

This section establishes the terminology and notation that will be used throughout the entire work, as well as the classification methodologies considered. The goal in classification is to assign an input vector $\mathbf{x}$ to a discrete label $y=C_{k}$, where $k \in$ $\{1, \ldots, K\}$. All the experiments in this paper cover the binary classification case, where $k \in\{+,-\}$, "-" representing graft failure and "+" a successful transplant. A formal framework for the semi-supervised learning problem could be introduced by considering a set of $l$ labelled points $L=\left(\mathbf{x}_{1}, y_{1}\right), \ldots,\left(\mathbf{x}_{l}, y_{l}\right)$ and $u$ unlabelled points $U=\mathbf{x}_{l+1}, \ldots, \mathbf{x}_{l+u}$, where the labels in $L$ are known and the ones in $U$ are unknown. Typically, $l \ll u$. Let $n=l+u$ be the total number of data points.

In the next subsections, we describe different proposals for exploiting unsupervised information when predicting transplant failure. Firstly, two strategies for generating unsupervised data are introduced (which in most applications would be not necessary, given that unsupervised data would be already available). Although our study is focused on liver transplantation, the scheme for generating unsupervised information (using virtual pairs) can be applicable to other types of transplants, or even applications where the aim is to compute the long-term compatibility of two or more individual and separate entities (e.g. when trying to compute the response that a patient will have to different treatments, where the first entity would be the information of the patient and the second one the characteristics of the treatment). Then, two approaches are considered to exploit this unsupervised data: 1) the most common one where available unlabelled patterns are included in the classifier construction step, and 2) a new proposal where the aim is to label unlabelled patterns and complement the dataset with the data that belong to the minority class. Note that this second approach can be used in any semi-supervised and imbalanced environment to label minority class patterns and create a fully supervised dataset. All the methods take into account the imbalance nature of the dataset, where the number of failures is considerably lower than the number of successful transplants.

\subsection{Strategies to capture unsupervised data}

Two sources of unsupervised data are used in this paper. The former considers available transplants with an unknown outcome. The latter exploits transplants which have not been performed (i.e. what is called virtual donor-recipient pairs). The main hypothesis of this paper is that both sources of information help to improve the performance of standard supervised algorithms and lead to more robust models for imbalanced data (specially in the case of virtual pairs, where the number of unlabelled data would be much higher than for recent transplants). Finally, we also propose to exploit the severity information of the donors (for more details see Section 2.1.3) to obtain virtual pairs that better represent the minority class (taking therefore advantage of expert knowledge). 


\subsubsection{Recent transplants}

Unsupervised data correspond in this case to those matchings whose follow-up time post-transplant is not completed, because the outcome is costly in terms of time (i.e. we could exploit those transplants before including them as supervised knowledge, since we do not know the output of the transplant). Note also that it is generally easier to gather information about past transplants (donor and recipient characteristics) than to gather information about the post-transplant follow-up, because follow-up information differs from country to country, while donor and recipient information is stored similarly in all countries. Nonetheless, the number of transplants for which the outcome is still unknown is generally low. Therefore, the idea would be to combine this approach with other of the methods tested in this paper.

\subsubsection{Virtual donor-recipient pairs}

Apart from the traditional semi-supervised approach, where unlabelled data is usually available, we consider that there are other applications where semi-supervised knowledge can be extracted from what we denominate as "potential" or "virtual" patterns. These patterns represent potential situations that have not occurred. In this case, unsupervised data makes use of nonreal transplants (based however, on real individuals). This is, we take into account potential organ transplantations that could have occurred in the past (but have not). To do so, if a given donor $D_{i}$ was originally assigned to recipient $R_{i}$ we consider all the patterns (or potential transplantations) joining $D_{i}$ and $\left\{R_{1}, \ldots, R_{i-1}, R_{i+1}, \ldots, R_{l}\right\}$. Note that this idea is applicable to other fields in which each pattern is composed of a set of individual units.

In other words, unlabelled data $U$ is extracted from different donor-recipient combinations from $L$. For example, consider the situation in which the transplant unit had to evaluate two recipients $R_{1}$ and $R_{2}$ for a given donor $D_{1}$. For some reason, the unit transplant decided that $R_{1}$ was more suitable for the organ (and this was the pair registered in the dataset). However, there exists a potential allocation $\left(D_{1}, R_{2}\right)$, which was not performed and for which we cannot possibly know the outcome of the procedure. This potential assignment can be used to complement the model in a semi-supervised manner (since it is not totally synthetic, but based on individual and real-world entities). Summarising, the unlabelled set of data is formed of virtual patterns, which we obtain by rotating donors and recipients.

For the ease of understanding, see Figure 2, where this idea is represented. The original matchings are used as supervised knowledge and the synthetic or virtual ones as unsupervised data to complement the dataset. Our hypothesis is that this information would not only help to improve the general performance of the base supervised classifier, but also the classification of minority classes, whose low representability results in a low classification rate (sometimes leading to a trivial classifier). Note that the number of generated unsupervised pairs depends on the number of labelled ones (if $l$ is the number of labelled patterns, we would have $l \cdot(l-1)$ unlabelled pairs). This makes necessary the use of large-scale and linear algorithms or methods for pattern selection. In our opinion, the inclusion of these virtual pairs should not introduce noise into the model, given that they represent an allocation that could have taken place (all of their values being real). These virtual allocations were not performed by the transplant team. This could be because, according to the different scores or their medical expertise they were not appropriate (meaning that they could have resulted in graft loss). However, they could have been very helpful to better represent the minority class, improving its separation.

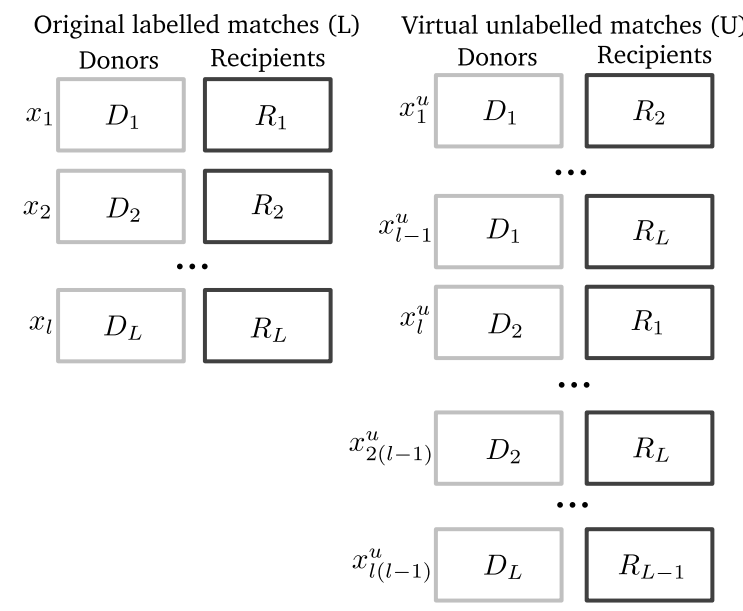

Figure 2: Graphical representation of the matching performed: original matches (obtained from the dataset) and virtual ones (generated rotating donors and recipients). Two main ideas are explored: whether donor-recipient compatibility can be predicted by machine learning and whether semi-supervised learning can help to improve this compatibility model.

We have compared three versions of this idea. The first one uses only $10 \%$ of the possible virtual pairs as unsupervised knowledge, the second one uses $50 \%$ of the data, while the last one uses all the data (100\%). This comparison intends to analyse the influence of the quantity of unsupervised information in the classifier performance.

\subsubsection{Use of extended donors}

The previous section is based on the idea that virtual semisupervised learning could contribute not only to the global correct classification rate but also to the discrimination of minority classes. However, it is clear that the discrimination of these minority classes would improve depending on the quality and quantity of unlabelled patterns that hypothetically belong to those classes. Because of this, we also propose the use of expert knowledge (in this case in the form of extended donors) as part of all virtual unlabelled patterns. Extended donors present at least two characteristics that are considered of risk and usually lead to a higher probability of graft failure. These characteristics are: extreme values of age, high number of days in the intensive care unit or ICU, inotropic drug usage, high body mass index or BMI and high cold ischemia time. Thus, in this case, the number of generated virtual patterns would be much lower (as we only use the combination of extended donors with the rest of recipients), but, theoretically, the ratio of patterns from the non-survival class would be higher. Large scale methods are also preferred, given that the number of virtual patterns would also significant. 
This experiment analyses the influence of the ratio of patterns belonging to the minority class with respect to the majority one in a semi-supervised and highly imbalanced classification problem.

\subsection{Approaches to incorporate unsupervised data}

The unsupervised data generated with the strategies in Section 2.1 are now considered during the classifier construction to obtain more reliable models. Two different approaches are used for this purpose.

\subsubsection{Standard semi-supervised method}

First of all, we consider the common semi-supervised learning setting, where unsupervised data are incorporated into the learning process, without assuming labels for these data. As it is common in this paradigm, and given the large-scale nature of the experiments performed, a light reformulation of Support Vector Machines (SVMs), which is specially designed for large-scale semi-supervised learning, has been chosen (Sindhwani and Keerthi, 2006) for all the experiments. The reason for this is that SVMs have been tested to a great extent in the case of semi-supervised learning and that the large number of data generated by our proposals makes impossible the application of any method that has not been specifically designed for large-scale problems. Note that large scale learning is often realistic only in a semi-supervised setting where a small set of labelled examples is available together with a large collection of unlabelled data. Because of this, in many applications, linear classifiers are preferred, given their easy implementation and interpretability and their empirical performance.

Some details are now given about the SVM version considered (Sindhwani et al., 2006; Sindhwani and Keerthi, 2006). An intuitive approach for using unlabelled data is to treat unknown labels as additional optimisation variables. For margin-based loss functions, this can be done by attempting to learn lowdensity separators. However, this results in a hard optimisation problem when unlabelled data is abundant (transductive SVMs have been used for this purpose, although they are susceptible to local minima). Because of this, a previously proposed global optimisation framework is considered, which handles these issues. Deterministic annealing is used to simplify the problem which is parametrically deformed to the original hard formulation and where the minimisers are smoothly tracked (Sindhwani et al., 2006). This method has been also successfully reformulated for large-scale and sparse scenarios (Sindhwani and Keerthi, 2006).

For appropriate comparisons, in the experiments of this paper, we compare this semi-supervised and linear SVM approach (which we will refer as SS-LSVC) with the original supervised and linear version of SVM.

\subsubsection{Label propagation for minority-class over-sampling}

Label propagation is one of the most widely used methods in semi-supervised applications (Zhou et al., 2004). Labels are propagated from the labelled patterns to the unlabelled ones making use of a neighbourhood graph. Note that, this approach presents three advantages: 1) a semi-supervised classification strategy is not needed, since the main idea is that the labelled set is augmented using unlabelled data, 2) non-linear classification methods can be used (as the number of training patterns does not grow to such an extent) and 3) this technique is applicable to all imbalanced semi-supervised scenarios in which we can label data for the minority class and include it as supervised knowledge.

Usually, a label propagation method present two problems in an application like the one considered. Firstly, when the class distribution is skewed, propagated labels would perpetuate this imbalance. Secondly, since the label propagation step involves an iterative process (or matrix operations including an inversion), its cost can be very high when a large number of patterns is available. These two issues make the application of the original label propagation scheme impossible. To solve this, we propose a two-step technique, that first selects potential patterns for the minority class using a neighbourhood analysis and afterwards applies the label propagation scheme. By doing this, we solve the high computational cost and minimize the bias towards the majority class (i.e. we select those patterns with the highest probability of belonging to the minority class).

We propose a new method based on label propagation. The strategy involves two steps, in order to alleviate the computational cost of the procedure and to ensure that selected patterns are from the minority class:

1. Selection of potential patterns for the minority class: Given the high computational cost of the label propagation method when a large number of patterns is available, the well-known $k$-nearest neighbour ( $k$-nn) method is chosen for performing a pre-selection of the patterns. In this sense, the patterns in $L$ (set of labelled data) are used to classify the patterns in $U$ (set of unlabelled data) using a $k$-nn approach, and those classified as minority class ones are selected for the next step of the procedure (represented by $P$ ). This step facilitates the computation of the label propagation method.

2. Label propagation step: The method in (Zhou et al., 2004) is used to propagate the labels of labelled patterns $L$ to the pre-selected unlabelled ones $P$. From this step, a matrix containing the probability that each pattern has of belonging to each class is obtained. The patterns in $P$ with the highest probability of belonging to the minority class are chosen and included as supervised data in the minority class (i.e. included in the set $L$ as new labelled data). In this case, the final number of patterns chosen is the one needed to balance the pattern distribution.

This method can be seen as a similar approach to oversampling and sample selection techniques, since new data are obtained and labelled by studying the neighbourhood of supervised data, which is common in both settings. In this case, instead of generating new synthetic data from a combination of patterns, we select those unlabelled patterns with a high probability of belonging to the minority class (or we under-sample potential data from the majority class). The most widely used over-sampling approach has been included in the experiments 
for comparison purposes. Linear and non-linear SVM methods are tested, in this case, using the new augmented labelled set.

\section{Experimental study and results}

In this section, the experiments are described and the results are discussed. A complete description of the liver transplantation dataset is firstly given, followed by a presentation of the relation of the methods to be compared, the measures evaluated and the experimental design. Finally, two different experiments are conducted to analyse the goodness of the proposed semisupervised approaches. The first experiment considers the application of semi-supervised learning using recent transplants as unsupervised information. The second experiment considers the use of virtual donor-recipient pairs, where new unlabelled data are generated, mixing donor and recipient pairs and creating new matches not present in the original dataset.

\subsection{Dataset description}

A retrospective analysis of an English liver transplant unit (King's College Hospital, in the United Kingdom) was made. Recipient and donor characteristics were reported at the time of transplant. Patients undergoing partial, split or living-donor liver transplantation and those undergoing combined or multivisceral transplants were excluded from the study. All patients were followed from the date of transplant until either death, or graft loss prior to one year after transplantation. Only those pairs with recipients over eighteen years of age between January 2002 and December 2010 were included. Thus, a dataset containing 822 donor-recipient pairs was collected. Several variables were selected: 16 recipient variables, 17 donor variables and 4 surgically related variables (which, for convenience, will be included in the donor and recipient groups depending on their meaning). The variables selected for the dataset can be seen in Table 1.

Every procedure, including obtaining informed consent, was conducted in accordance with the ethical standards of the local Human Research Ethics Committee and in accordance with the ethical standards of the 1975 Declaration of Helsinki.

Note that 3 of the transplant variables were included in the donor set of characteristics (combined transplant, complete or partial graft and cold ischemia time) because they are related to and depend primarily on the donor. The same applies to the multi-organ harvesting variable, which is included in the recipient set of features.

For our study, we consider two datasets (each one representing a different period of time to control graft failure). More specifically, we consider graft failure before 3 months (KC3M dataset) and before 12 months (KC12M). The choice of class limits for the dataset ( 3 and 12 months) has been made by experts as the most pertinent (being these considered for previous studies in Cruz-Ramírez et al. (2012); Briceño et al. (2014); Pérez-Ortiz et al. (2014)).

Note that, even under the case that the model derived would be successful, it would not be universal, but rather a demonstra-
Table 1: Principal characteristics of the dataset: features considered, number of patterns and classes, etc.

\begin{tabular}{|c|c|c|}
\hline \multicolumn{3}{|c|}{$\begin{array}{l}\text { Number of patterns: } 822 \text {, number of classes: } 2 \text {, number of features: } 37 \text {, class distribution: } \\
\{81,741\}(\mathrm{KC} 3 \mathrm{M}),\{112,710\}(\mathrm{KC} 12 \mathrm{M})\end{array}$} \\
\hline Atribute name & Type & Value \\
\hline \multicolumn{3}{|c|}{ Recipient (R) } \\
\hline Age (A-R) & Numeric & {$[18,76]$} \\
\hline Gender (G-R) & Binary & $0=$ male $; 1=$ female \\
\hline Body mass index (BMI-R) & Numeric & {$[26,68.3]$} \\
\hline Diabetes mellitus (DM-R) & Binary & $0=$ absence $; 1=$ presence \\
\hline Arterial hypertension (AH-R) & Binary & $0=$ absence $; 1=$ presence \\
\hline Dialysis at transplant (DT-R) & Binary & $0=$ absence $; 1=$ presence \\
\hline Etiology $(E-R)$ & Nominal & $\begin{array}{l}0=\text { virus } \mathrm{C} \text { cirrhosis; } 1=\text { alcohol; } 2=\text { virus } \\
\mathrm{B} \text { cirrhosis; } 3=\text { fulminant hepatic failure; } 4= \\
\text { primary biliary cirrhosis; } 5=\text { primary scleros- } \\
\text { ing cholangitis; } 6=\text { others }\end{array}$ \\
\hline Portal thrombosis (PT-R) & Ordinal & $0=$ no $; 1=$ partial $; 2=$ complete \\
\hline Waiting list time (WLT-R) & Numeric & {$[0,1021]$} \\
\hline MELD (inclusion) (MI-R) & Numeric & {$[1,46]$} \\
\hline MELD (at transplant) (MT-R) & Numeric & {$[6,50]$} \\
\hline TIPS at transplant (TT-R) & Binary & $0=$ absence $; 1=$ presence \\
\hline Hepatorrenal syndrome (HS-R) & Binary & ; $1=$ presence \\
\hline Upper abdominal surgery (UAS-R) & Binary & $0=$ absence $; 1=$ presence \\
\hline $\begin{array}{l}\text { Pretransplant status performance } \\
\text { (PSP-R) }\end{array}$ & Nominal & $\begin{array}{l}0=\text { at home; } 1=\text { hospitalized; } 2=\text { hospitalized } \\
\text { in ICU; } 3=\text { hospitalized in ICU with mechan- } \\
\text { ical ventilation }\end{array}$ \\
\hline Cytomegalovirus (C-R) & Binary & $0=$ absence $; 1=$ presence \\
\hline Multi-organ harvesting (MOH-R) & Binary & $0=$ no $; 1=$ yes \\
\hline \multicolumn{3}{|c|}{ Donor $(\mathrm{D})$} \\
\hline Age (A-D) & Numeric & {$[11,86]$} \\
\hline Gender (G-D) & Binary & $0=$ male $; 1=$ female \\
\hline Body mass index (BMI-D) & Numeric & {$[14.38,53.35]$} \\
\hline Diabetes mellitus (DM-D) & Binary & nce; 1 = presence \\
\hline Arterial hypertension (AH-D) & Binary & $0=$ absence $; 1=$ presence \\
\hline Cause of exitus (CE-D) & Nominal & $\begin{array}{l}0=\text { brain trauma; } 1=\text { cerebral vascular acci- } \\
\text { dent; } 2=\text { anoxia; } 3=\text { deceased vascular after } \\
\text { cerdiac death; } 4=\text { others }\end{array}$ \\
\hline \multicolumn{3}{|c|}{$\begin{array}{l}\text { Hospitalization length in ICU (HL- Numeric }[0,58] \\
\text { D) }\end{array}$} \\
\hline Hypotension episodes (HE-D) & Binary & $0=$ absence $; 1=$ presence \\
\hline High inotropic drug use (HIDU-D) & Binary & $0=$ absence $; 1=$ presence \\
\hline Creatinine plasma level (CPL-D) & Numeric & {$[0.1,9.5]$} \\
\hline Sodium plasma level (SPL-D) & Numeric & {$[123,181]$} \\
\hline \multicolumn{3}{|c|}{$\begin{array}{l}\text { Aspartate transaminase level (ATL- Numeric }[1,1090] \\
\text { D) }\end{array}$} \\
\hline \multicolumn{3}{|c|}{$\begin{array}{l}\text { Alanine aminotransferase plasma Numeric }[2,974] \\
\text { level (AAPL-D) }\end{array}$} \\
\hline Total bilirubin (TB-D) & Numeric & {$[0.1,3.4]$} \\
\hline Hepatitis B (HB-D) & Binary & $0=$ absence $; 1=$ presence \\
\hline Hepatitis C (HC-D) & Binary & $0=$ absence $; 1=$ presence \\
\hline Cytomegalovirus (C-D) & Binary & $0=$ absence $; 1=$ presence \\
\hline Combined transplant (CT-D) & Binary & $0=$ no $; 1=$ yes \\
\hline Complete or partial graft (CPG-D) & Binary & $0=$ no; $1=$ yes \\
\hline Cold ischemia time (CIT-D) & Ordinal & $0=<6 \mathrm{~h} . ; 1=6-12 \mathrm{~h} . ; 2=>12 \mathrm{~h}$. \\
\hline The end-point variable is the time le & ling up to & liver failure. We consider two cases: 1) Befor \\
\hline
\end{tabular}

tion that machine learning techniques can be used to derive a donor-recipient matching model ${ }^{1}$.

\subsection{Methods compared}

Different methods have been compared for this study, some of them specially indicated for imbalanced classification. On the one hand, the following supervised methods are used, based only on the original supervised information:

\footnotetext{
${ }^{1}$ The next issue being how to create a supranational model or how to train the model using the information of each liver transplantation unit
} 
- SVC and LSVC: Non-linear and linear versions of the well-known Support Vector Classifier (SVC) (Cortes and Vapnik, 1995; Chang and Lin, 2011).

- CS-SVC and CS-LSVC: Non-linear and linear versions of the cost-sensitive SVC (Chang and Lin, 2011), where different class-weights are used for imbalanced classification (i.e. a higher cost is set for minority classes based on the imbalance ratio). In this way, the weights are set according to the ratio of minority class patterns with respect to the majority class ones.

- SVC+SMOTE and LSVC+SMOTE: Non linear and linear versions of SVC combined with a standard over-sampling approach (Chawla et al., 2002), which is one of the most popular techniques for approaching imbalanced classification. The number of synthetic patterns to be generated is that needed to balance the class distribution.

- MPENSGA2-E and MPENSGA2-MS: Neural network models obtained from the Pareto front built by a multiobjective evolutionary algorithm (MPENSGA2). The first model is the extreme corresponding to maximum entropy (obtaining optimal values in Accuracy), while the second model is the extreme corresponding to maximum minimum sensitivity. Both models are used in Cruz-Ramírez et al. (2012) as a decision support system for donorrecipient matching in liver transplantation, in such a way that the probability of belonging to the survival class is maximised and the probability of belonging to the nonsurvival class is minimised.

On the other hand, the following semi-supervised methods are used, based on both the original supervised data and unsupervised sources of information:

- SS-LSVC: Semi-supervised linear version of SVC (presented in Section 2.2.1) using unsupervised patterns. For the first experiment, unsupervised patterns come from unlabelled data (as explained in Section 2.1.1, typically, because the transplant is very recent, and the outcome is yet unknown), while, for the second experiment, the unsupervised data is synthetically generated as virtual donorrecipient pairs (as explained in Section 2.1.2).

- SS-LSVC-Ext: Semi-supervised linear version of SVC using only extended donors for the computation of the synthetic unsupervised data (see Section 2.1.3). As stated before, these donors present a series of characteristics that are considered of risk and usually lead to a higher probability of graft failure, which makes it interesting for improving the classification of the minority class. Extended criteria donors are those that present at least two of the following restrictions: age $>75$ years; hospitalisation length in ICU $>4$ days; high inotropic drug use $=1$; $\mathrm{BMI}>30$; Cold Ischemia Time $=2$ ( $>12$ hours).

- SVC-LP and LSVC-LP: Non-linear and linear versions of SVC using the label propagation strategy for labelling unsupervised synthetic patterns and use them as supervised ones (Section 2.2.2). More specifically, the label from supervised patterns is propagated to unsupervised patterns. Then, those examples with a higher probability of belonging to the minority class are chosen and labelled (with the label of the minority class). After the pattern selection process, the standard SVC strategy is considered, but, in this case, the class distribution is balanced. Note that, from all possible unsupervised virtual pairs, as many patterns as needed to balance the distribution are labelled.

\subsection{Evaluation metrics}

Several metrics can be considered for evaluating classifiers. The most common one in machine learning is the Accuracy (Acc) or correct classification rate. However, this measure is not the best option for some classification scenarios (e.g. in the presence of class imbalance). Because of this, we complement accuracy (Acc) with the geometric mean of the sensitivities $(G M)$, which is specially designed for imbalanced classification (López et al., 2013). The metrics used can be defined as follows:

- Acc: The correct classification rate or accuracy is the ratio of correctly classified patterns:

$$
A c c=\frac{1}{N} \sum_{i=1}^{N}\left(I\left(y_{i}^{*}=y_{i}\right)\right),
$$

where $I(\cdot)$ is the zero-one loss function, $y_{i}$ is the desired output for pattern $i, y_{i}^{*}$ is the prediction of the model, and $N$ is the total number of patterns in the dataset. Acc values vary from 0 to 1 , and it represents the global performance in the classification task. This metric has many disadvantages, specially when imbalanced problems are considered.

- $G M$ : The geometric mean of the sensitivities is an average of the percentage of the correct classification of each of the classes:

$$
G M=\sqrt[K]{\prod_{k=1}^{K} S_{k},}
$$

where $S_{k}=\frac{1}{N_{k}} \sum_{i=1}^{N_{k}}\left(I\left(y_{i}^{*}=y_{i}\right)\right)$ is the sensitivity of the $k$-th class, i.e. the percentage of patterns correctly predicted as belonging to the $k$-th class with respect to the total number of examples in this class. For all the datasets considered in this paper, we have $K=2$, i.e. two classes, graft survival and failure.

The joint use of the Acc and GM metrics is difficult, because $A c c$ is usually opposite to any metric designed for imbalanced learning (Fernández-Caballero et al., 2010; Soda, 2011). Detecting the minority class often comes at the expense of a decrease in global accuracy, which is the price to pay for a nontrivial classifier. The combination of both metrics helps then to detect trivial classifiers (i.e. where the same label is predicted for all inputs) and supports the individual classification rate of both classes, as well as the global accuracy. A balance 
between these two metrics means that 1) the classifier is accurate on the whole and 2) it distinguishes successfully between the classes of the problem. If we exclusively focus on the minority class, we could introduce other type of error (the one where a safe transplant is classified as problematic). Because of this, we consider the use of the $G M$ metric, that takes all the classes in the problem into account, aiming at a good result on the whole. However, this error is still possible, and because of this, the allocation system should take into account other factors, e.g. that no recipient remains indefinitely in the waiting list.

\subsection{Experiment configuration}

For the evaluation of the results, a stratified 10-fold technique has been applied to divide the data. The results are taken as the mean and standard deviation of the two metrics for the 10 test sets.

The parameters of each algorithm were chosen using a 5fold nested validation with each of the 10 training sets. Although $G M$ seems to be the most appropiate metric for this problem, both options were considered. In this way, the final parameter combination was the one resulting in the highest Acc or $G M$ in the validation set (as can be checked in Table 3), so that the importance of considering the $G M$ in this application can be analysed. The kernel selected for all the non-linear kernel methods is the Gaussian one, $K(\mathbf{x}, \mathbf{y})=\exp \left(-\frac{\|\mathbf{x}-\mathbf{y}\|^{2}}{\sigma^{2}}\right)$, where $\sigma$ is the kernel width. The kernel width was selected within the values $\left\{10^{-3}, 10^{-2}, \ldots, 10^{3}\right\}$, as was the cost parameter $C$ associated to all supervised SVM methods (linear and non-linear versions). The $k$ parameter associated to the $k$-nn (for SVC+SMOTE, LSVC+SMOTE, SVC-LP and LSVC-LP) was also cross-validated in the range $\{1,3,5\}$. For all the methods using large-scale semi-supervised SVMs Sindhwani and Keerthi (2006), the regularisation parameters $w$ and $u$ were optimised within the values $\left\{10^{-4}, 10^{-3}, 10^{-2}, 10^{-1}, 10^{0}\right\}$.

The same parameter configuration as in Cruz-Ramírez et al. (2012) has been used for the multi-objective evolutionary methods MPENSGA2-E and MPENSGA2-MS.

\subsection{Experimental results}

Finally, the experiments are presented and discussed in this section. Statistical tests are performed to determine the significance of the differences observed.

\subsubsection{First experiment}

In order to check whether real unlabelled data (coming from, for example, recent transplants) can be beneficial for model construction, the first experiment considers the labels of a given percentage of training data, using the rest as unsupervised information. The results are included in Table 2, where the effect of the amount of supervised information is studied. Both supervised and semi-supervised approaches are compared using a linear model (LSVC and SS-LSVC). The percentage of supervised data varies from $2.5 \%$ to $50 \%$. The first method (LSVC) is the linear version of SVM using the specified percentage of supervised patterns. The second method (SS-LSVC) is also a linear version of the semi-supervised approach of SVM and
Table 2: Results of the first experiment: Mean \pm Standard deviation results obtained (Acc and GM for the test sets) for LSVC and SS-LSVC.

\begin{tabular}{cccc}
\hline \hline \multicolumn{4}{c}{ Graft survival at 3 months: KC3M } \\
\hline Sup. data & Method & Acc & $G M$ \\
\hline \multirow{2}{*}{$2.5 \%$} & LSVC & $\mathbf{8 9 . 1 7} \pm \mathbf{2 . 1 1}$ & $3.44 \pm 10.87$ \\
& SS-LSVC & $86.13 \pm 2.95$ & $\mathbf{1 0 . 1 9} \pm \mathbf{1 6 . 4 1}$ \\
\hline \multirow{2}{*}{$5 \%$} & LSVC & $\mathbf{8 7 . 4 6} \pm \mathbf{4 . 2 8}$ & $6.80 \pm 14.34$ \\
& SS-LSVC & $85.65 \pm 3.32$ & $\mathbf{1 7 . 7 2} \pm \mathbf{2 3 . 2 6}$ \\
\hline \multirow{2}{*}{$10 \%$} & LSVC & $\mathbf{7 9 . 6 8} \pm \mathbf{6 . 7 5}$ & $28.05 \pm 22.65$ \\
& SS-LSVC & $79.55 \pm 5.15$ & $\mathbf{3 0 . 3 5} \pm \mathbf{2 4 . 2 7}$ \\
\hline \multirow{2}{*}{$30 \%$} & LSVC & $82.12 \pm 3.82$ & $28.38 \pm 26.35$ \\
& SS-LSVC & $\mathbf{8 2 . 1 3} \pm \mathbf{4 . 9 9}$ & $\mathbf{3 4 . 9 0} \pm \mathbf{2 2 . 3 8}$ \\
\hline \multirow{2}{*}{$50 \%$} & LSVC & $\mathbf{8 8 . 2 0} \pm \mathbf{2 . 7 3}$ & $10.19 \pm 16.41$ \\
& SS-LSVC & $84.91 \pm 5.23$ & $\mathbf{2 6 . 2 5} \pm \mathbf{2 4 . 9 3}$ \\
\hline \hline \multirow{4}{*}{$2.5 \%$} & Graft surVival at 12 months: KC12M \\
& LSVC & $\mathbf{8 5 . 2 8} \pm \mathbf{2 . 2 6}$ & $2.91 \pm 9.19$ \\
& SS-LSVC & $80.16 \pm 4.91$ & $\mathbf{1 1 . 5 3} \pm \mathbf{1 9 . 2 0}$ \\
\hline \multirow{2}{*}{$5 \%$} & LSVC & $\mathbf{8 0 . 7 7} \pm \mathbf{7 . 9 9}$ & $16.98 \pm 22.98$ \\
& SS-LSVC & $70.29 \pm 6.12$ & $\mathbf{3 9 . 7 3} \pm \mathbf{1 8 . 1 3}$ \\
\hline \multirow{2}{*}{$10 \%$} & LSVC & $68.36 \pm 4.71$ & $36.34 \pm 16.45$ \\
& SS-LSVC & $\mathbf{7 3 . 1 0} \pm \mathbf{5 . 2 8}$ & $\mathbf{4 1 . 4 4} \pm \mathbf{1 0 . 8 1}$ \\
\hline \multirow{2}{*}{$30 \%$} & LSVC & $78.83 \pm 4.49$ & $23.32 \pm 16.97$ \\
& SS-LSVC & $\mathbf{7 9 . 6 8} \pm \mathbf{3 . 6 9}$ & $\mathbf{3 4 . 8 5} \pm \mathbf{1 6 . 3 9}$ \\
\hline \multirow{2}{*}{$50 \%$} & LSVC & $\mathbf{8 5 . 8 9} \pm \mathbf{1 . 5 3}$ & $0.00 \pm 0.00$ \\
& SS-LSVC & $80.40 \pm 4.89$ & $\mathbf{3 0 . 0 4} \pm \mathbf{1 2 . 7 8}$ \\
\hline \hline
\end{tabular}

The best result is in bold face.

uses the remaining data as unsupervised information to complement the learning.

As can be seen in Table 2 even when very few labelled patterns are used (e.g. consider the case of $10 \%$ of supervised data), the semi-supervised methodology obtains better results in terms of $G M$, aiming at a more balanced classification. Moreover, it generally maintains a relatively competitive Acc. These results highlight the importance of using all available information about transplants even when the label is unknown, i.e. when the follow-up time post-transplant has not been completed. The variances are, in general, very high, which is a common case when using the $G M$ metric, because of its definition. One of the conclusions that can be extracted from this is that the quantity of unlabelled patterns is not as important as the quality of those (which also justifies the high variance of the results and the difference between the percentages).

\subsubsection{Second experiment}

The second experiment complements the available supervised information with the construction of virtual pairs. In this case, the number of unlabelled patterns to include in the model for SS-LSVC is set to $10 \%, 50 \%$ and $100 \%$ of all possible pairs $^{2}$, in order to analyse the effect of this number in the per-

\footnotetext{
${ }^{2}$ Note that the number of virtual pairs is $l \cdot(l-1)$ (i.e. in this case using the $90 \%$ of the data for train we obtain around 560.000 unlabelled pairs).
} 
formance of the method. The results of all the methods for this experiment are included in Table 3. From these results, some conclusions can be drawn, but in order to do so, the imbalanced nature of the dataset must be taken into account (i.e. the need to correctly classify the minority class). It is well-known that $A c c$ presents some problems when dealing with imbalanced data, thus the $G M$ metric (or a balance between both of them) should be the priority.

From the results of this second experiment (Table 3), it can be seen that the models with the highest accuracy are trivial models (i.e., they ignore the classification of the minority class, i.e. $G M=0 \%)$. As seen, this metric is also essential in the crossvalidation process, leading to more fair models. As stated before, to represent successfully the good performance of a classifier, we need, in this case, a balance between Acc and GM. Note that two classifiers could have the same value on $G M$ but very different $A c c$, and the same Acc but different GM. GM does not take the number of patterns that compose each class into account, whereas Acc does. For example, we could have a classifier that classifies correctly a $75 \%$ of the minority class and only $15 \%$ of the majority one. In this case, these measures would be approximately $G M=33.5 \%$ and $A c c=20 \%$. However, if we switch the class percentages, we would have $G M=33.5 \%$ and $A c c=69 \%$. Different errors are committed in each case. However, in abscence of specific costs for the problem considered in this paper, we set the same costs for each type of errors: 1) type I error, where a safe transplant is said to be problematic, and 2) type II error, where a problematic transplant is predicted to be safe. Both cases could be said to be equally important, because a type I error may result in deaths in the waiting list, while type II errors may end with graft loss. It can be seen that using this measure, the non-linear SVC model is capable of achieving more balanced classifiers (reaching results of $G M$ of $11 \%$ and $13 \%$ for $\mathrm{KC} 3 \mathrm{M}$ and $\mathrm{KC} 12 \mathrm{M}$ respectively). Nonetheless, the linear version (LSVC) does not distinguish both classes successfully.

Concerning cost-sensitive approaches (CS-SVC and CSLSVC), these models show a better performance in the minority class, at the cost, however, of sacrificing Acc (obtaining in some cases results of $A c c$ around or lower than $50 \%$, a result similar to that of a random classifier). The models based on over-sampling (SVC+SMOTE and LSVC+SMOTE) present better performance in general, which is consistent with the findings of the literature (Galar et al., 2012), where it is shown that over-sampling is generally preferred to non sampling strategies. However, again, the linear version does not present satisfactory results.

Comparing semi-supervised approaches to supervised ones, one can observe that the virtual simulation of patterns is a helpful tool for stabilizing the model and for the correct classification of the minority class. A reason for this could be that this technique generates more incompatible donor-recipient pairs that would not be considered in real life when using the MELD score or the knowledge of the medical community, and this helps the classification of the minority class (in a similar manner to over-sampling).

Moreover, the use of additional data (even when it is unsuper- vised) simplifies the classification task and alleviates the imbalance problem (which, as seen in previous works, does not only depends on the imbalance ratio but also on the number of patterns, Barandela et al. (2004); Japkowicz and Stephen (2002b)). Therefore, it could be said that the use of this virtual information (without any restriction, i.e. the methods SS-LSVC-10\%, SS-LSVC-50\% and SS-LSVC-100\%) results generally in more robust and accurate models, at both $\mathrm{KC} 3 \mathrm{M}$ and $\mathrm{KC} 12 \mathrm{M}$.

The number of semi-supervised data (i.e. SS-LSVC-10\%, SS-LSVC-50\% and SS-LSVC-100\%) is a significant parameter, leading to different results in each case. In particular, it seems that the choices of the patterns to be included are in some cases more important than the number of patterns itself (at least to improve the classification of the minority class), although a more stable solution is obtained when using all data (a better trade-off between Acc and GM). Recall that the semisupervised models used in this case are all linear (given the large amount of unsupervised patterns used). However, these models obtain better results that other non-linear models that only consider labelled data. Concerning the results of specially designed methods for imbalanced learning (i.e. CS-SVC and SVC+SMOTE and their variants), these do not obtain a good balance between the two metrics, at opposed to our semisupervised approaches. It can be inferred then that the used of virtual pairs (composed of real individual entities) is preferable than synthetic over-sampling.

The use of extended donors for generating the synthetic semisupervised pairs (SS-LSVC-Ext method) also improves the stability of the final model (achieving promising balance between the two metrics). This could mean that the ratio of unlabelled data from each class is also a determining factor when approaching a problem with semi-supervised learning. In this case, the ratio could be more equitable, given the use of extended donors, which have been linked in the literature to a higher probability of graft failure. Our last approaches (SVCLP and LSVC-LP) make use of a label propagation technique for obtaining a new set of supervised data (only for the minority class) to complement the dataset. These methodologies obtain the models that could be consider as the best ones for this application. In this case, linear models obtain very similar performance to non-linear ones, which shows that the inclusion of semi-supervised information and unlabelled data can help to simplify the decision boundaries.

Finally, we compare our results to MPENSGA2-E and MPENSGA2-MS (Cruz-Ramírez et al., 2012), which are the ones previously used in the literature for solving the same problem of donor-recipient matching. Two acceptable models are obtained for both methods, the former focusing on global accuracy and the latter on the accuracy for all classes, which could be then jointly used as a decision support system. Comparing, for example, LSVC-LP and MPENSGA2-MS, it can be appreciated that a better trade-off can be expected from our semisupervised approach (i.e. better results in terms of Acc for similar $G M$ ), even when the models are linear (such as LSVC-LP). This has to be taken into account together with the fact that our algorithms are simpler and can be applied to a wide range of semi-supervised and supervised algorithms. 
Table 3: Results of the second experiment: Mean \pm Standard deviation results obtained (Acc and GM for the test sets) for the different methodologies considered. The effect of the cross-validation metric used for optimising the parameters is also studied (C. Metric).

\begin{tabular}{|c|c|c|c|}
\hline \multicolumn{4}{|c|}{ Graft survival at 3 months: $\mathrm{KC} 3 \mathrm{M}$} \\
\hline Method & C. Metric & $A c c$ & $G M$ \\
\hline SVC & $A c c$ & $90.15 \pm 0.35$ & $0.00 \pm 0.00$ \\
\hline SVC & $G M$ & $82.72 \pm 2.22$ & $11.57 \pm 14.93$ \\
\hline LSVC & $A c c$ & $90.15 \pm 0.35$ & $0.00 \pm 0.00$ \\
\hline LSVC & $G M$ & $90.15 \pm 0.35$ & $0.00 \pm 0.00$ \\
\hline CS-SVC & $A c c$ & $86.38 \pm 0.44$ & $0.00 \pm 0.00$ \\
\hline CS-SVC & $G M$ & $68.36 \pm 4.54$ & $36.99 \pm 18.36$ \\
\hline CS-LSVC & $A c c$ & $30.63 \pm 28.61$ & $19.56 \pm 18.38$ \\
\hline CS-LSVC & $G M$ & $48.43 \pm 11.65$ & $49.57 \pm 8.19$ \\
\hline SVC+SMOTE & $G M$ & $81.63 \pm 3.00$ & $34.83 \pm 28.88$ \\
\hline LSVC+SMOTE & $G M$ & $55.38 \pm 22.21$ & $40.77 \pm 19.78$ \\
\hline MPENSGA2-E & - & $89.29 \pm 1.76$ & $7.02 \pm 14.81$ \\
\hline MPENSGA2-MS & - & $59.26 \pm 6.65$ & $47.79 \pm 20.86$ \\
\hline \multicolumn{4}{|c|}{ Semi-supervised approaches } \\
\hline SS-LSVC-10\% & $G M$ & $79.07 \pm 4.73$ & $40.33 \pm 24.52$ \\
\hline SS-LSVC-50\% & $G M$ & $69.59 \pm 5.92$ & $52.41 \pm 16.88$ \\
\hline SS-LSVC-100\% & $G M$ & $80.53 \pm 4.06$ & $41.02 \pm 25.09$ \\
\hline SS-LSVC-Ext & $G M$ & $80.05 \pm 3.73$ & $47.85 \pm 21.77$ \\
\hline SVC-LP & $G M$ & $76.16 \pm 3.02$ & $\mathbf{5 0 . 3 5} \pm \mathbf{1 5 . 0 4}$ \\
\hline LSVC-LP & $G M$ & $78.47 \pm 2.90$ & $49.99 \pm 21.26$ \\
\hline Best Model LSVC-LP & $G M$ & 76.83 & 76.01 \\
\hline \multicolumn{4}{|c|}{ Graft survival at 12 months: KC12M } \\
\hline Method & C. Metric & $A c c$ & $G M$ \\
\hline SVC & $A c c$ & $86.25 \pm 0.53$ & $0.00 \pm 0.00$ \\
\hline SVC & $G M$ & $86.99 \pm 2.28$ & $13.65 \pm 17.64$ \\
\hline LSVC & $A c c$ & $86.38 \pm 0.44$ & $0.00 \pm 0.00$ \\
\hline LSVC & $G M$ & $86.38 \pm 0.44$ & $0.00 \pm 0.00$ \\
\hline CS-SVC & $A c c$ & $90.15 \pm 0.35$ & $0.00 \pm 0.00$ \\
\hline CS-SVC & $G M$ & $56.82 \pm 6.88$ & $55.09 \pm 9.10$ \\
\hline CS-LSVC & $A c c$ & $47.73 \pm 33.63$ & $18.70 \pm 19.00$ \\
\hline CS-LSVC & $G M$ & $51.48 \pm 18.18$ & $43.76 \pm 18.86$ \\
\hline SVC+SMOTE & $G M$ & $77.85 \pm 3.07$ & $30.73 \pm 23.40$ \\
\hline LSVC+SMOTE & $G M$ & $51.73 \pm 14.00$ & $48.28 \pm 11.66$ \\
\hline MPENSGA2-E & - & $85.16 \pm 1.47$ & $5.81 \pm 12.27$ \\
\hline MPENSGA2-MS & - & $59.38 \pm 7.39$ & $50.57 \pm 10.88$ \\
\hline \multicolumn{4}{|c|}{ Semi-supervised approaches } \\
\hline SS-LSVC-10\% & $G M$ & $68.02 \pm 6.87$ & $45.94 \pm 13.57$ \\
\hline SS-LSVC-50\% & $G M$ & $76.28 \pm 5.07$ & $40.84 \pm 8.55$ \\
\hline SS-LSVC-100\% & $G M$ & $77.49 \pm 4.50$ & $39.52 \pm 7.96$ \\
\hline SS-LSVC-Ext & $G M$ & $77.00 \pm 4.30$ & $44.59 \pm 12.39$ \\
\hline SVC-LP & $G M$ & $71.29 \pm 2.30$ & $52.74 \pm 11.80$ \\
\hline LSVC-LP & $G M$ & $69.58 \pm 3.16$ & $49.12 \pm 11.18$ \\
\hline Best Model LSVC-LP & $G M$ & 75.90 & 63.36 \\
\hline
\end{tabular}

The best result is in bold face and the second best result is in italics.

Note that the best models for LSVC-LP have been also included in Table 3, both showing outstanding results. Since these models are linear, the weights can be used for interpretability purposes and will be later studied.

Now, we study whether the differences found in both experiments are significant. Each pair of algorithms has been compared by means of the non-parametric and signed-rank Wilcoxon test (Wilcoxon, 1945), using the 10 results of the 10 -fold design. A popular way to compare the overall performances of algorithms is to count the number of cases on which an algorithm is the overall winner. Using this test, each pair of methods was compared for each dataset (KC3M and $\mathrm{KC} 12 \mathrm{M}$ ) and the total number of statistically significant wins or losses was recorded, together with the number of draws (or absence of statistically significant differences). For the first experiment, the number of of wins, draws and losses is 10: we compare five versions of the dataset $(2.5 \%, 5 \%, 10 \%, 30 \%$ and $50 \%)$ with two different configurations for the target variable (KC3M and $\mathrm{KC} 12 \mathrm{M})$. For the second experiment, 34 comparisons are included ( 2 configurations for the target variable and 17 methods to compare each method against). A level of significance of $\alpha=0.10$ has been considered. The results of these tests for Acc and $G M$ are shown in Table 4, where the number of wins (W), draws (D) and loses (L) is shown.

In relation to the first experiment, the best results in $G M$ are obtained by the semi-supervised approach, while the supervised method shows improvements in Acc. However, these good results in Acc are obtained at the cost of performing trivial classifications in some cases, as shown in Table 2.

For the second experiment, semi-supervised approaches are the ones which obtain the best balance between wins in Acc and wins in $G M$ (e.g. analyse the results of SS-LSVC-100\%, SS-LSVC-Ext and SVC-LP). Supervised standard SVM approaches (e.g. SVC and LSVC) obtain the best results in term of Acc but the worst results for $G M$, which shows again the difficulty in optimising both metrics at the same time without specific methods. Cost-sensitive and over-sampling based techniques seem to perform better (specially in the case of CS-SVC and CS-LSVC cross-validated by $G M$ ). However, their number of wins for Acc is still relatively low in comparison to the semisupervised methods. A great difference can be observed between cost-sensitive and over-sampling based approaches when cross-validated by the two metrics, which shows the need of properly tuning the parameters for the methods used. In relation with MPENSGA2-based methods, MPENSGA2-E does not obtain acceptable results in terms of $G M$, as opposed to MPENSGA2-MS (which obtains comparable results in $G M$ to our proposals SVC-LP and LSVC-LP, but with worse results for $A c c$ ). Concerning the quantity of unlabelled data, it can be seen that more data results in a more robust model, without explicitly harming any of the selected metrics. Finally, it can be seen that both linear and non-linear models based on label propagation (i.e. SVC-LP and LSVC-LP) obtain similar performance in statistical terms, so that we can conclude that unlabelled data complements perfectly the supervised data and leads to more easily separable decision regions.

In general, the following ideas can be extracted from our experiments: 1) The quantity of unsupervised patterns is crucial, but the quality of those is also of vital importance. In the case of an imbalanced distribution, expert knowledge can make the difference. 2) When the set of data is large, non-linear methods do not show a great improvement over linear ones. 3) Virtual pairs (based on real data) help to improve the performance of machine learning methods in the detection of minority examples to a greater extent than totally synthetic information. 4) Labelling unsupervised data to augment the proportion of supervised patterns is a simple and suitable strategy that shows very promising results, helping to achieve a trade-off between both metrics.

In order to analyse the most important variables for predict- 
Table 4: Wilcoxon statistical test results (W or wins, D or draws and L or loses) for the different experiments considered. Both datasets ( $\mathrm{KC} 3 \mathrm{M}$ and $\mathrm{KC} 12 \mathrm{M})$ are considered for these results.

\begin{tabular}{cccc}
\hline \hline Metric & \multicolumn{4}{c}{$A c c$} & $G M$ \\
\hline Method & C. Metric & $\mathrm{W} / \mathrm{D} / \mathrm{L}$ & $\mathrm{W} / \mathrm{D} / \mathrm{L}$ \\
\hline \multicolumn{4}{c}{ First experiment } \\
\hline LSVC & $G M$ & $\mathbf{5} / \mathbf{4} / \mathbf{1}$ & $0 / 7 / 3$ \\
SS-LSVC & $G M$ & $1 / 4 / 5$ & $\mathbf{3} / \mathbf{7} / \mathbf{0}$ \\
\hline \multicolumn{4}{c}{ Second experiment } \\
\hline SVC & $A c c$ & $27 / 6 / 1$ & $0 / 10 / 24$ \\
SVC & $G M$ & $24 / 0 / 10$ & $0 / 12 / 22$ \\
LSVC & $A c c$ & $27 / 6 / 1$ & $0 / 10 / 24$ \\
LSVC & $G M$ & $\mathbf{3 1 / 3 / 0}$ & $0 / 10 / 24$ \\
CS-SVC & $A c c$ & $27 / 6 / 1$ & $0 / 10 / 24$ \\
CS-SVC & $G M$ & $4 / 3 / 27$ & $19 / 11 / 4$ \\
CS-LSVC & $A c c$ & $0 / 9 / 25$ & $9 / 6 / 19$ \\
CS-LSVC & $G M$ & $1 / 5 / 28$ & $16 / 17 / 1$ \\
SVC+SMOTE & $G M$ & $17 / 5 / 12$ & $12 / 14 / 8$ \\
LSVC+SMOTE & $G M$ & $1 / 6 / 27$ & $15 / 17 / 2$ \\
MPENSGA2-E & - & $26 / 3 / 5$ & $0 / 11 / 23$ \\
MPENSGA2-MS & - & $3 / 4 / 27$ & $18 / 16 / 0$ \\
SS-LSVC-10\% & $G M$ & $11 / 5 / 18$ & $14 / 17 / 3$ \\
SS-LSVC-50\% & $G M$ & $12 / 6 / 16$ & $16 / 14 / 4$ \\
SS-LSVC-100\% & $G M$ & $15 / 7 / 12$ & $15 / 15 / 4$ \\
SS-LSVC-Ext & $G M$ & $15 / 6 / 13$ & $16 / 17 / 1$ \\
SVC-LP & $G M$ & $10 / 4 / 20$ & $\mathbf{1 9 / 1 5 / 0}$ \\
LSVC-LP & $G M$ & $9 / 8 / 17$ & $18 / 16 / 0$ \\
\hline \hline
\end{tabular}

ing graft survival after transplantation we study the influence of each variable in the best model (using SVC-LP) in Table 3 at 3 months after transplantation (KC3M). The linear weights obtained are included in Table 5. Note that all the binary variables are included in this case (54 variables that are the result of decomposing the original 37 ones). The variables have been ranked considering the absolute value of their weights. Note that the variables can have a positive or negative impact on the output (where the survival at 3 months represent the positive class). The bias of the model is -0.985 . It can be seen that the most influential variables are mostly the ones related to the recipient, although there are some variables related to the donor that also have an impact on the model (thus justifying the need to use both sources of information). The most important variables for the characterisation of the survival are: arterial hypertension (recipient), pretransplant status performance (recipient), MELD (at transplant), diabetes mellitus (recipient), recipient etiology (virus), gender (recipient), cause of exitus of donor (anoxia), tips at transplant (recipient) and other factors such as age and body mass index (recipient). This shows that although the MELD variable has an influence in our model (being an important factor) there are other variables with greater or similar impact. These findings are consistent with the results reported in literature where the age is an important factor contributing to the donor risk index. Similarly, prolonged ICU hospitalization is a strong predictor of early graft dysfunction and poor initial functioning that increased post-transplant hospital costs.

\section{Discussion and proposed system for organ allocation}

Donor-recipient (D-R) matching is performed at the moment of organ procurement. However, since MELD does not consider donor characteristics, the organ is assigned to the patient listed first on the list of the most ill, a strategy that cannot be truly considered as a matching. Therefore, using MELD a con-
Table 5: Best model weights per variable (graft survival prediction at 3 months).

\begin{tabular}{cccccc}
\hline \hline Rank & Binary variable & Weight & Rank & Binary variable & Weight \\
\hline 1 & AH-R & -0.429 & 28 & CIT-D=0 & -0.089 \\
2 & PSP-R=3 & 0.325 & 29 & A-D & -0.088 \\
3 & MT-R & 0.307 & 30 & PSP-R=2 & 0.088 \\
4 & DM-R & -0.293 & 31 & HC-D & 0.083 \\
5 & PSP-R=0 & -0.279 & 32 & CIT-D=1 & -0.078 \\
6 & E-R=3 & -0.246 & 33 & CPG-D & 0.074 \\
7 & G-R & 0.233 & 34 & HS-R & 0.064 \\
8 & CE-D=2 & -0.209 & 35 & HB-D & -0.054 \\
9 & TT-R & -0.191 & 36 & CE-D=1 & -0.051 \\
10 & A-R & 0.191 & 37 & HIDU-D & -0.041 \\
11 & BMI-R & 0.183 & 38 & PSP-R=1 & -0.039 \\
12 & C-R & -0.175 & 39 & BMI-D & 0.033 \\
13 & UAS-R & 0.170 & 40 & DT-R & -0.033 \\
14 & E-R=0 & 0.148 & 41 & SPL-D & 0.032 \\
15 & CE-D=3 & -0.144 & 42 & AAPL-D & -0.031 \\
16 & MI-R & 0.126 & 43 & G-D & 0.021 \\
17 & E-R=6 & 0.115 & 44 & AH-D & 0.020 \\
18 & E-R=2 & -0.109 & 45 & ATL-D & 0.017 \\
19 & E-R=1 & 0.107 & 46 & WLT-R & -0.015 \\
20 & PT-R=0 & 0.106 & 47 & CPL-D & -0.011 \\
21 & PT-R=1 & -0.106 & 48 & E-R=5 & -0.007 \\
22 & CIT-D=2 & 0.106 & 49 & DM-D & 0.006 \\
23 & HL-D & 0.094 & 50 & CT-D & 0.003 \\
24 & E-R=4 & -0.094 & 51 & HE-D & 0.003 \\
25 & CE-D=4 & 0.093 & 52 & CE-D=0 & 0.000 \\
26 & TB-D & -0.091 & 53 & C-D & 0.000 \\
27 & MOH-D & -0.089 & 54 & PT-R=2 & 0.000 \\
\hline \hline
\end{tabular}

crete D-R combination does not necessarily result in the best combination in terms of utility. Because of this, and based on the best model obtained in the previous subsection, a novel liver allocation system is proposed. The first stage of the system is the selection of the first $k$-recipients on the waiting list (i.e. the $k$ sickest patients, since patients on the waiting list are sorted according to the MELD score). In case of draws, the time spent on the waiting list is considered. After this, graft survival after transplantation is predicted for these $k$ recipients using the best model obtained in our experiments. Then, the organ is assigned to the recipient whose predicted survival is the highest ( in this case, we only consider two classes, but the probability of belonging to the survival class could be considered for a finer grain discrimination). In case of draws between two or more recipients, the one with the highest MELD is selected. This new system complements the assignment of the MELD score, by taking into account donor and operative factors. Figure 3 underlines the general ideas of the proposed liver allocation system.

Input

1. Waiting_List: Characteristics of patients in waiting list

2. Organ_Ch: Characteristics of the organ to be allocated

3. Best_Model: Computed allocation model

Output

1. Final_Recipient: Selected recipient for the organ allocation

Procedure

1. Initialise the number of patients to consider $(k)$

2. Sorted_List: Sort Waiting_List by MELD and waiting list time

3. Potential_recipients: Select first k recipients of Sorted_list

4. Survival_Response: Predict output for Potential_Recipients using Best_Model and Organ_Ch

5. Best_Recipients: Select recipients associated to the highest Survival_Response

6. MELD_List: Get MELD (from Waiting_List) for Best_Recipients

7. Final_Recipient: Select the patient from Best_Recipients with the highest MELD

Figure 3: Pseudocode of the proposed liver allocation system. 
A simulation of our proposed system (and of the two best models obtained in this paper) is included in Table 8 where the recipients listed in Table 6 and the donors in Table 7 are used. The result of each combination of pairs donor-recipient is evaluated with the best models and the output of the system is included in the Table (- if the matching belongs to the nonsurvival class and + otherwise). The number of times that each recipient and organ results in survival is also reported in the Table. Several conclusions can be extracted from these results. Firstly, it can be seen that both models (at 3 and 12 months) are robust and agree in the output of the transplant in most cases. Note that, the only incongruence that these two models show is when the model at 3 months predict that the match will lead to graft failure but the model at 12 months predict that the match will be successful. This is because the rest of options (e.g. that the model at 3 months predicts survival and the model at 12 months predicts non-survival) are viable options. Moreover, this incongruence only occurs in $6 \%$ of the matches. On the other hand, it can be seen that the MELD score is in some cases related to the output of the transplant, meaning this that recipients with a low MELD (as the situation 1 tested) have in general a satisfactory post-transplant output and potential incompatibilities (i.e. the probability of graft failure) increases with the MELD range (e.g. compare the number of graft loses for recipients with MELD $<20$ and MELD > 30). This fact motivates the use of an allocation system that predicts match compatibilities, which is vital to maximize the organ utility. As can be seen, our model will agree with the MELD score in the vast majority of situations, however, it will also help the medical community to detect situations in which the allocation is not secure.

Concerning extended donors, the one that represents the most secure approach is D1 (resulting in graft loss only 2 times). It can be seen that this donor, compared to the rest, presents a low age and a normal BMI, it does not present diabetes mellitus, arterial hypertension or hepatitis and the cold ischemia time is medium. On the other hand, D3 is the one that results in nonsurvival more times. This donor presents diabetes mellitus, arterial hypertension, cytomegalovirus and a high cold ischemia time. Furthermore, comparing D2 and D4 it can be seen that D2 results in predicted survival more times than D4 (despite the age of the donors). D4 is only 26 years old but has a relatively low BMI and diabetes mellitus. This donor was also 7 days in the intensive care unit and the organ has a high cold ischemia time. In relation to non-extended donors, the one that present the highest survival rate are D7 and D8 (which have very low cold ischemia time and do not present diabetes, arterial hypertension or cytomegalovirus, variables that have been shown to be discriminative in the model interpretation phase). The one that has the lowest failing rate is D10.

Concerning the recipients, there are many of them that have a high survival rate. However, the one which could be most interesting is $\mathrm{R} 13$, which has a $100 \%$ survival rate but belongs to the group of recipients with MELD > 30. The main difference with the rest of recipients of the group is the etiology, the lack of diabetes mellitus, cytomegalovirus, hepatorrenal syndrome and the pretransplant state (at home against at ICU with mechanical ventilation).

Table 6: Characteristics of the recipients selected from the dataset used to test the allocation system.

\begin{tabular}{l|ccccccccccccccccc}
\hline \hline Rec. & A & G & BMI & DM & AH & DT & E & PT & WLT & MI & MT & TT & HS & UAS & PSP & C & MOH \\
\hline R1 & 69 & 1 & 40.06 & 1 & 1 & 0 & 6 & 1 & 357 & 11 & 18 & 0 & 0 & 1 & 0 & 1 & 0 \\
R2 & 51 & 0 & 29.86 & 1 & 0 & 0 & 0 & 0 & 48 & 15 & 15 & 0 & 0 & 0 & 0 & 1 & 0 \\
R3 & 71 & 0 & 30.52 & 0 & 0 & 0 & 5 & 0 & 18 & 12 & 12 & 0 & 0 & 0 & 1 & 1 & 0 \\
R4 & 18 & 1 & 38.10 & 0 & 0 & 0 & 6 & 0 & 344 & 6 & 8 & 0 & 0 & 0 & 0 & 0 & 1 \\
R5 & 42 & 0 & 32.65 & 0 & 0 & 0 & 0 & 0 & 628 & 4 & 6 & 0 & 0 & 0 & 0 & 0 & 1 \\
R6 & 37 & 1 & 35.43 & 0 & 0 & 0 & 3 & 0 & 3 & 25 & 29 & 0 & 0 & 0 & 3 & 1 & 0 \\
R7 & 57 & 1 & 40.06 & 0 & 1 & 1 & 6 & 0 & 768 & 24 & 26 & 0 & 0 & 0 & 0 & 1 & 0 \\
R8 & 27 & 0 & 37.18 & 0 & 0 & 0 & 6 & 0 & 84 & 25 & 24 & 0 & 0 & 1 & 0 & 1 & 0 \\
R9 & 47 & 0 & 33.03 & 0 & 1 & 0 & 6 & 1 & 34 & 23 & 23 & 1 & 0 & 1 & 0 & 1 & 0 \\
R10 & 50 & 1 & 47.56 & 1 & 0 & 0 & 4 & 1 & 477 & 13 & 22 & 0 & 0 & 0 & 0 & 1 & 0 \\
R11 & 34 & 0 & 27.70 & 0 & 0 & 0 & 3 & 0 & 5 & 43 & 50 & 0 & 0 & 0 & 3 & 1 & 0 \\
R12 & 39 & 1 & 32.65 & 1 & 0 & 0 & 3 & 1 & 1 & 34 & 40 & 0 & 0 & 0 & 3 & 1 & 0 \\
R13 & 37 & 0 & 37.18 & 0 & 0 & 0 & 0 & 0 & 70 & 35 & 36 & 1 & 0 & 0 & 0 & 0 & 1 \\
R14 & 22 & 1 & 44.44 & 0 & 0 & 0 & 3 & 0 & 1 & 30 & 33 & 0 & 0 & 0 & 3 & 0 & 1 \\
R15 & 66 & 0 & 31.92 & 1 & 1 & 0 & 5 & 0 & 270 & 27 & 31 & 0 & 1 & 0 & 0 & 1 & 0 \\
R16 & 19 & 1 & 34.60 & 0 & 0 & 0 & 3 & 0 & 2 & 25 & 27 & 0 & 0 & 0 & 3 & 0 & 1 \\
R17 & 57 & 1 & 44.44 & 0 & 0 & 0 & 4 & 0 & 10 & 28 & 27 & 0 & 1 & 0 & 0 & 0 & 0 \\
R18 & 68 & 0 & 31.92 & 0 & 0 & 0 & 6 & 0 & 297 & 8 & 27 & 0 & 0 & 0 & 0 & 1 & 0 \\
R19 & 64 & 0 & 38.10 & 0 & 1 & 0 & 6 & 0 & 413 & 27 & 27 & 0 & 0 & 0 & 0 & 1 & 1 \\
R20 & 46 & 0 & 30.86 & 1 & 0 & 0 & 3 & 0 & 3 & 24 & 27 & 0 & 0 & 0 & 3 & 1 & 0 \\
\hline \hline
\end{tabular}

arterial hypertension; DT: dialysis at transplant; E: etiology; PT: portal thrombosis; WLT: waiting list time; MI: MELD score at listing; MT: MELD score at transplant; TT: TIPS at transplant; HS: hepatorrenal syndrome; UAS: upper abdominal surgery; PSP: status

performance pretransplant; $\mathrm{C}$ : cytomegalovirus; $\mathrm{MOH}$ : Multi-organ harvesting.

Note that at the moment of the implementation of this allocation system more conservative factors can be introduced. For example, the probability of graft survival can be computed (e.g. in SVM using the distance of the projected pattern to the bias) and used for the system considering a statistical test that gives us information about significant differences in the probabilities. By doing this, the organ would be assigned to the recipient with the highest probability of survival (given that the difference in probability with respect to the rest of recipients is significantly higher). If this is not the case, the MELD score should be considered. On the other hand, a constraint can be included in the system so that no recipient remains forever in the waiting list. To do so, a formulation that considers the number of times that the recipient was selected in the $k$ first recipients but not selected (i.e. the times where the recipient was considerably ill but not allocated an organ) can be implemented. Finally, as a clinical test of our proposal is not feasible, we propose to analyse firstly our methodology in a more controlled environment comparing the choices of MELD and our system.

\section{Conclusions}

This paper studies different sources of unsupervised information to improve the performance of binary classification models for the problem of liver transplantation outcome prediction. The two sources identified are those transplants whose followup process has not been completed and virtual transplants combining donor and recipients which were not matched during the allocation. Unsupervised data are introduced in the learning process by considering two methods: standard semi-supervised 
Table 7: Donor and other surgery factors from the set of patterns used to test the allocation system.

\begin{tabular}{lcccccccccccccccccccc}
\hline \hline D. & A & G & BMI & DM & AH & CE & HL & HE & HIDU & CPL & SPL & ATL & AAPL & TB & HB & HC & C & CT & CPG & CIT \\
\hline D1(E) & 21 & 1 & 21.61 & 0 & 0 & 0 & 6 & 1 & 1 & 1.00 & 142 & 815.00 & 204 & 0.50 & 0 & 0 & 0 & 0 & 0 & 1 \\
D2(E) & 81 & 1 & 22.58 & 0 & 0 & 1 & 2 & 0 & 1 & 1.30 & 145 & 45.00 & 30 & 1.00 & 0 & 0 & 0 & 0 & 0 & 1 \\
D3(E) & 58 & 0 & 27.76 & 1 & 1 & 1 & 4 & 0 & 1 & 1.10 & 143 & 49.00 & 11 & 0.50 & 0 & 0 & 1 & 1 & 0 & 2 \\
D4(E) & 26 & 0 & 18.94 & 1 & 0 & 1 & 7 & 0 & 1 & 0.80 & 134 & 27.00 & 21 & 2.20 & 0 & 0 & 1 & 0 & 1 & 2 \\
D5(E) & 64 & 0 & 24.05 & 0 & 0 & 0 & 1 & 0 & 1 & 1.20 & 146 & 38.00 & 23 & 0.50 & 1 & 0 & 1 & 0 & 0 & 2 \\
D6 & 55 & 1 & 24.80 & 0 & 1 & 1 & 6 & 0 & 1 & 0.60 & 156 & 41.75 & 38 & 0.30 & 0 & 1 & 0 & 0 & 0 & 1 \\
D7 & 19 & 0 & 22.34 & 0 & 0 & 2 & 10 & 0 & 0 & 0.70 & 142 & 80.91 & 82 & 0.20 & 0 & 0 & 0 & 0 & 1 & 0 \\
D8 & 68 & 0 & 26.99 & 0 & 0 & 1 & 6 & 0 & 1 & 1.20 & 141 & 18.51 & 14 & 0.60 & 0 & 0 & 0 & 0 & 0 & 0 \\
D9 & 34 & 0 & 22.09 & 0 & 0 & 1 & 1 & 0 & 0 & 1.00 & 143 & 27.00 & 15 & 0.80 & 0 & 0 & 1 & 0 & 1 & 1 \\
D10 & 38 & 1 & 25.10 & 0 & 0 & 1 & 8 & 0 & 0 & 0.40 & 134 & 48.92 & 50 & 0.30 & 0 & 0 & 0 & 1 & 0 & 2 \\
\hline
\end{tabular}

Abbreviations: A: age; G: gender; BMI: body mass index; DM: diabetes mellitus; AH: arterial hypertension; CE: cause of exitus; HL: hospitalisation length in intensive care unit; HE: hypotension episodes > $1 \mathrm{hr}<60 \mathrm{mmHg}$; HIDU: high inotropic drug use; CPL: creatinine plasma level; SPL: sodium plasma level; ATL: aspartate transaminase level; AAPL: alanin aminotransferase plasma level; TB: total bilirubin; HB: hepatitis B (core Ab positive); HC: hepatitis C (positive serology); C: cytomegalovirus; CT: combined transplant; CPG: complete or partial graft; CIT: cold ischemia time.

learning and a specific label propagation scheme. The imbalanced nature of the datasets (where the number of failures is significantly lower than that of successful transplants) is taken into account, both during synthetic unsupervised data generation and during the label propagation step. Supervised methods (including both standard classifiers and different mechanisms for imbalanced data) are compared against our proposals, using a dataset from the liver transplantation unit of the King College's hospital (UK), where two different versions of the binary problem are considered (predicting the graft failure after 3 months or after 12 months).

The results obtained show that the imbalanced nature of the dataset must be taken into account in order to avoid trivial classifiers, and that the popular techniques used for dealing with imbalanced distributions (such as cost-sensitive learning or oversampling methods) do not obtain acceptable results. However, we show how the use of unsupervised data (real or virtual) results in more robust and fair models, both for the minority and the majority class, and how, the proportion of majority/minority examples and the quality of those could be more important than the quantity itself. The label propagation method is found to focus consistently on the minority class virtual pairs. Moreover, by forcing the virtual pairs to include extended criteria donors, the results are also improved. Concerning the simulation of the proposed system, it can be seen that our model agrees to a large extent with the MELD score, but can also help to detect incompatibilities between donors and recipients.

Future work comprises the extension of this semi-supervised classification idea to the multiclass approach (where a finer grain classification is performed) and to other hospitals with the aim of constructing a supranational model.

\section{Acknowledgment}

This work has been partially subsidized by the TIN201454583-C2-1-R and the TIN2015-70308-REDT projects of the Spanish Ministerial Commission of Science and Technology (MINECO, Spain), FEDER funds (EU), the P11-TIC-7508 project of the "Junta de Andalucía" (Spain), the PI-0312-2014 project of the "Fundación pública andaluza progreso y salud"
(Spain) and the PI15/01570 project ("Proyectos de Investigación en Salud"). The authors M. Pérez-Ortiz, P.A. Gutiérrez and M.D. Ayllón-Terán have contributed equally to the preparation of this paper.

\section{References}

Barandela, R., Valdovinos, R.M., Sánchez, J.S., Ferri, F.J., 2004. The imbalanced training sample problem: Under or over sampling?, in: Fred, A.L.N., Caelli, T., Duin, R.P.W., Campilho, A.C., de Ridder, D. (Eds.), SSPR/SPR, Springer. p. 806

Briceño, J., Cruz-Ramírez, M., Prieto, M., Navasa, M., de Urbina, J.O., Orti, R., Gómez-Bravo, M.A., Otero, A., Varo, E., Tomé, S., Clemente, G., nares, R.B., Bárcena, R., Cuervas-Mons, V., Solórzano, G., Vinaixa, C., Rubn, A., Colmenero, J., Valdivieso, A., Ciria, R., Hervás-Martínez, C., de la Mata, M., 2014. Use of artificial intelligence as an innovative donor-recipient matching model for liver transplantation: Results from a multicenter spanish study. Journal of Hepatology 61, 1020 - 1028.

Briceño, J., Solorzano, G., Pera, C., 2000. A proposal for scoring marginal liver grafts. Transplant International 13, S249-S252.

Busuttil, R.W., Tanaka, K., 2003. The utility of marginal donors in liver transplantation. Liver Transplant 9, 651-663.

Cai, D., He, X., Han, J., 2007. Semi-supervised discriminant analysis, in: Computer Vision, 2007. ICCV 2007. IEEE 11th International Conference on, pp. $1-7$.

Chang, C.C., Lin, C.J., 2011. Libsvm: A library for support vector machines. ACM Trans. Intell. Syst. Technol. 2, 27:1-27:27.

Chawla, N.V., Bowyer, K.W., Hall, L.O., Kegelmeyer, W.P., 2002. Smote: Synthetic minority over-sampling technique. Journal of Artificial Intelligence Research 16, 321-357.

Cohen, I., Cozman, F.G., Sebe, N., Cirelo, M.C., Huang, T.S., 2004. Semisupervised learning of classifiers: theory, algorithms, and their application to human-computer interaction 26, 1553- 1566.

Cortes, C., Vapnik, V., 1995. Support-vector networks. Machine Learning 20, 273-297.

Cruz-Ramírez, M., Hervás-Martínez, C., Fernandez-Caballero, J., Briceño, J., de la Mata, M., 2012. Multi-Objective Evolutionary Algorithm for DonorRecipient Decision System in Liver Transplants. European Journal of Operational Research 222, 317-327.

Dutkowski, P., Oberkofler, C., Slankamenac, K., Puhan, M., Schadde, E., Mllhaupt, B., Geier, A., Clavien, P., 2011. Are there better guidelines for allocation in liver transplantation? A novel score targeting justice and utility in the model for end-stage liver disease era. Annals of Surgery 254, 745-753.

Feng, S., Goodrich, N., Bragg-Gresham, J., Dykstra, D., Punch, J., DebRoy, M., Greenstein, S., Merion, R., 2006. Characteristics associated with liver graft failure: The concept of a donor risk index. American Journal of Transplantation 6, 783-790.

Fernández-Caballero, J.C., Martínez-Estudillo, F.J., Hervás-Martínez, C., Gutiérrez-Peña, P.A., 2010. Sensitivity Versus Accuracy in Multiclass Prob- 
Table 8: Simulation of the proposed system, where different situations are considered. The predicted values of the system are reported, where - corresponds to the non-survival class (at 3 or 12 months) and + to the survival class. The number of times that an organ or a recipient results in a survival output is also included to ease the analysis.

\begin{tabular}{|c|c|c|c|c|c|c|c|c|c|c|c|c|c|c|c|c|c|c|c|c|c|c|c|c|}
\hline \multirow[b]{2}{*}{ Rec.(MELD) } & \multicolumn{12}{|c|}{ Survival at 3 months } & \multicolumn{12}{|c|}{ Survival at 12 months } \\
\hline & D1 & $\mathrm{D} 2$ & D3 & D4 & D5 & D6 & D7 & D8 & D9 & D10 & Total $(+)$ & Rec.(MELD) & D1 & D2 & D3 & D4 & D5 & $\mathrm{D}$ & $6 \mathrm{D}$ & D7 1 & D8 & D9 & D10 & Total $(+)$ \\
\hline R1(18) & + & + & + & + & + & + & + & + & + & + & 10 & $\mathrm{R} 1(18)$ & + & + & - & + & + & + & + & + & + & + & + & 9 \\
\hline $\mathrm{R} 4(8)$ & + & + & + & + & + & + & + & + & + & + & 10 & $\mathrm{R} 4(8)$ & + & + & + & + & + & + & + & + & + & + & + & 10 \\
\hline R5(6) & + & + & + & + & + & + & + & + & + & + & 10 & $\mathrm{R} 5(6)$ & + & + & + & + & + & + & + & + & + & + & + & 10 \\
\hline \multicolumn{25}{|c|}{ Situation 2: recipients with MELD values between 20 and 30} \\
\hline $\mathrm{R} 8(24)$ & + & + & - & - & + & + & + & + & - & - & 6 & $\mathrm{R} 8(24)$ & + & + & - & + & + & + & + & + & + & + & - & 8 \\
\hline $\mathrm{R} 9(23)$ & + & + & + & + & + & + & + & + & + & + & 10 & $\mathrm{R} 9(23)$ & + & + & + & + & + & + & + & + & + & + & + & 10 \\
\hline $\mathrm{R} 10(22)$ & + & + & + & + & + & + & + & + & + & + & 10 & $\mathrm{R} 10(22)$ & + & + & + & + & + & + & + & + & + & + & + & 10 \\
\hline \multicolumn{25}{|c|}{ Situation 3: recipients with MELD values greater than 30} \\
\hline R11(50) & + & - & - & - & + & - & - & - & - & - & 2 & $\mathrm{R} 11(50)$ & + & - & - & - & - & - & + & + & + & - & - & 3 \\
\hline $\mathrm{R} 12(40)$ & + & + & + & + & + & + & + & + & + & + & 10 & $\mathrm{R} 12(40)$ & + & - & - & - & - & - & + & + & + & - & - & 3 \\
\hline R17(27) & + & + & - & - & + & + & + & + & - & ـ & 6 & R17 & - & - & - & - & - & - & - & - & - & - & - & 0 \\
\hline $\mathrm{R} 18(27)$ & + & + & + & + & + & + & + & + & + & + & 10 & & + & + & + & + & + & + & + & + & + & + & + & 10 \\
\hline R19(27) & + & + & + & + & + & + & + & + & + & + & 10 & R19(27) & + & + & + & + & + & + & + & + & + & + & + & 10 \\
\hline R20(27) & + & + & + & + & + & + & + & + & + & + & 10 & R20(27) & + & + & + & + & + & + & + & + & + & + & + & 10 \\
\hline Total(+) & 20 & 17 & 14 & 14 & 20 & 17 & 17 & 18 & 14 & 14 & & Total(+) & 18 & 16 & 11 & 13 & 15 & 16 & 6 & 19 & 19 & 15 & 12 & \\
\hline
\end{tabular}

lems Using Memetic Pareto Evolutionary Neural Networks. IEEE Transacctions on Neural Networks 21, 750-770.

Galar, M., Fernández, A., Barrenechea, E., Bustince, H., Herrera, F., 2012. A Review on Ensembles for the Class Imbalance Problem: Bagging-, Boosting-, and Hybrid-Based Approaches. Systems, Man, and Cybernetics, Part C: Applications and Reviews, IEEE Transactions on 42, 463-484.

He, H., García, E.A., 2009. Learning from imbalanced data. IEEE Trans. on Knowl. and Data Eng. 21, 1263-1284.

Hernández-González, J., Inza, I., Lozano, J.A., 2016. Weak supervision and other non-standard classification problems: a taxonomy. Pattern Recognition Letters 69, $49-55$.

Huang, T.M., Kecman, V., 2004. Knowledge-Based Intelligent Information and Engineering Systems: 8th International Conference, KES 2004, Wellington, New Zealand, September 20-25, 2004, Proceedings, Part III. Springer Berlin Heidelberg, Berlin, Heidelberg. chapter Semi-supervised Learning from Unbalanced Labeled Data - An Improvement. pp. 802-808.

Japkowicz, N., Stephen, S., 2002a. The class imbalance problem: A systematic study. Intelligent Data Analysis 6, 429-449.

Japkowicz, N., Stephen, S., 2002b. The class imbalance problem: A systematic study. Intell. Data Anal. 6, 429-449.

Kamath, P., Kim, W., 2007. The Model for End-stage Liver Disease (MELD). Hepatology 45, 797-805.

Li, S., Wang, Z., Zhou, G., Lee, S.Y.M., 2011. Semi-supervised learning for imbalanced sentiment classification, in: Proceedings of the Twenty-Second International Joint Conference on Artificial Intelligence - Volume Volume Three, AAAI Press. pp. 1826-1831.

López, V., Fernández, A., García, S., Palade, V., Herrera, F., 2013. An insight into classification with imbalanced data: Empirical results and current trends on using data intrinsic characteristics. Information Sciences 250, $113-141$.

Ma, Y., Luo, G., Li, J., Chen, A., 2011. Combating class imbalance problem in semi-supervised defect detection, in: Computational Problem-Solving (ICCP), 2011 International Conference on, pp. 619-622.

Maalouf, M., Siddiqi, M., 2014. Weighted logistic regression for large-scale imbalanced and rare events data. Knowledge-Based Systems 59, $142-148$.

Pérez-Ortiz, M., Cruz-Ramírez, M., Ayllón-Terán, M., Heaton, N., Ciria, R., Hervás-Martínez, C., 2014. An organ allocation system for liver transplantation based on ordinal regression. Applied Soft Computing 14, 88 - 98

Pérez-Ortiz, M., Gutiérrez, P.A., Tino, P., Hervás-Martínez, C., 2016. Oversampling the minority class in the feature space. IEEE Transactions on Neural
Networks and Learning Systems 27, 1947-1961.

Rana, A., Hardy, M.A., Halazun, K.J., Woodland, D.C., Ratner, L.E., Samstein, B., Guarrera, J.V., Brown, R.S., Emond, J.C., 2008. Survival outcomes following liver transplantation (SOFT) score: a novel method to predict patient survival following liver transplantation. American Journal of Transplantation 8, 2537-46.

Schaubel, D.E., Guidinger, M.K., Biggins, S.W., Kalbfleisch, J.D., Pomfret, E.A., Sharma, P., Merion, R.M., 2009. Survival benefit-based deceaseddonor liver allocation. Am J Transplant 9, 970-81.

Sindhwani, V., Keerthi, S.S., 2006. Large scale semi-supervised linear svms, in: Proceedings of the 29th annual international ACM SIGIR conference on Research and development in information retrieval, ACM. pp. 477-484.

Sindhwani, V., Keerthi, S.S., Chapelle, O., 2006. Deterministic annealing for semi-supervised kernel machines, in: Proceedings of the 23rd international conference on Machine learning, ACM. pp. 841-848.

Soda, P., 2011. A multi-objective optimisation approach for class imbalance learning. Pattern Recognition 44, 1801-1810.

Su, C.J., Wu, C.Y., 2011. Jade implemented mobile multi-agent based, distributed information platform for pervasive health care monitoring. Applied Soft Computing 11, $315-325$.

Tseng, M.H., Liao, H.C., 2009. The genetic algorithm for breast tumor diagnosis - the case of dna viruses. Applied Soft Computing 9, 703 - 710.

Wilcoxon, F., 1945. Individual comparisons by ranking methods. Biometrics Bulletin 1, 80-83.

Zhou, D., Bousquet, O., Lal, T.N., Weston, J., Schölkopf, B., 2004. Learning with local and global consistency, in: Thrun, S., Saul, L.K., Schölkopf, B. (Eds.), Advances in Neural Information Processing Systems 16. MIT Press, pp. 321-328.

Zhou, L., 2013. Performance of corporate bankruptcy prediction models on imbalanced dataset: The effect of sampling methods. Knowledge-Based Systems 41, $16-25$.

Zhu, X., 2005. Semi-Supervised Learning Literature Survey. Technical Report 1530. Computer Sciences, University of Wisconsin-Madison. URL: http: //pages.cs.wisc.edu/ jerryzhu/pub/ssl_survey.pdf. 